\title{
Estimación de la biomasa aérea de Eucalyptus globulus Labill plantado en cercos vivos, distrito Huertas, Junín (Perú)
}

\author{
Above-ground biomass estimation of Eucalyptus globulus Labill \\ in living fences, Huertas district, Junín (Peru)
}

Jean C. Valverde ${ }^{1}$, Víctor M. Barrena ${ }^{2, *}$ y Roxana Guillén ${ }^{2}$

Recibido: 29 enero 2019 | Aceptado: 05 mayo 2019 | Publicado en línea: 30 junio 2019 Citación: Valverde, JC; Barrena, VM; Guillén, R. 2019. Estimación de la biomasa aérea de Eucalyptus globulus Labill plantado en cercos vivos, distrito Huertas, Junín (Perú). Revista Forestal del Perú 34(1): 52-65. DOI: http://dx.doi.org/10.21704/rfp.v34i1.1285

\begin{abstract}
Resumen
El objetivo de este trabajo fue determinar una ecuación para estimar la biomasa aérea de los árboles de Eucalyptus globulus Labill, plantados como cercos vicos en el distrito de Huertas, provincia de Jauja del departamento de Junín. Para lo cual se tomó una muestra de 40 árboles con diámetro a la altura del pecho superior a los $15 \mathrm{~cm}$. Se utilizó la metodología del muestreo destructivo, separando cada componente del árbol y tomando muestras para su análisis en el laboratorio. Se elaboró la ecuación de biomasa aérea basada en el método de mínimos cuadrados, así como el de los mínimos cuadrados ponderados; para ello se utilizaron los datos tomados en el campo. Los modelos previamente seleccionados fueron sometidos a pruebas estadísticas con la finalidad de identificar el modelo que muestra mejores resultados. Estas pruebas fueron: prueba de bondad de ajuste, donde todos los modelos presentaron $\mathrm{R}^{2}$ superior al 80 por ciento; pruebas de significancia Fisher y t-student, aquellos modelos que no cumplían con la significancia del modelo o uno de sus variables fueron descartados; el Análisis de Residuos se llevó de forma gráfica, las ecuaciones que no cumplían con los supuestos de homocedasticidad de errores, fueron ponderados hasta lograr el ajuste adecuado, que cumpla con el supuesto mencionado. Finalmente, se calculó el índice de Furnival (IF) para modelos ponderados, la ecuación del modelo 8 , cuya variable independiente es dap ${ }^{2} h^{2}$ fue la que presentó el menor valor de IF y fue seleccionada como la ecuación con el mejor ajuste y precisión para estimar la biomasa aérea de árboles de Eucalyptus globulus en cercos vivos.
\end{abstract}

Palabras clave: Biomasa aérea, ecuación de biomasa, Análisis de Regresión Ponderada, cercos vivos, Eucalyutus globulus Labill

\footnotetext{
${ }^{1}$ World Agroforestry Centre, Latin America Office, Lima, Perú.

${ }^{2}$ Departamento Académico de Manejo Forestal, Facultad de Ciencias Forestales, Universidad Nacional Agraria La Molina (UNALM), Av. La Molina s/n, La Molina, Lima, Perú.

* Autor de Correspondencia: vbarrena@lamolina.edu.pe
} 


\begin{abstract}
The objective of this research was to determine an equation for the estimation of aerial biomass of Eucalyptus globulus Labill trees planted as a living fence in the district of Huertas province of Jauja in the department of Junín, from a sample of 40 trees with a diameter at breast height greater than $15 \mathrm{~cm}$ was used. The destructive sampling methodology was used, separating each component of the tree and taking samples for analysis in the laboratory. The methodology used to construct the aerial biomass equation from field data was based on linear regression using the method of least squares and weighted least squares. The previously selected models were submitted to statistical tests in order to identify the model that shows best results. These tests were: goodness of fit test, where all models had $\mathrm{R}^{2}$ above $80 \%$; Fisher and t-student significance tests, those models that did not meet the significance of the model or one of its variables were discarded; Analysis of residues was carried out graphically, those equations that did not comply with the assumption of homoscedasticity of errors, were weighted until an adequate adjustment was achieved, that meets the aforementioned assumption. Finally, the Furnival index (IF) for weighted equations was calculated, the equation of model 8, whose independent variable is $\mathrm{dap}^{2} \mathrm{~h}^{2}$ was the one that had the lowest IF value and was selected as the equation with the best fit and precision to estimate of the aerial biomass for the Eucalyptus globulus planted in living fence.
\end{abstract}

Key words: Aerial biomass, biomass equation, weighted regression analysis, living fences, Eucalyutus globulus Labill

\section{Introducción}

El Eucalyptus globulus Labill (Myrtacae) es la especie forestal de mayor producción en la sierra central desde alrededor de 100 años. Según el MINAG (2013), para el 2012 se tuvo una producción a nivel nacional de $68089,26 \mathrm{~m}^{3}$ de madera aserrada y $526350 \mathrm{~m}^{3}$ de madera rolliza. Es una especie con buenas características para el uso estructural siempre que se realice un manejo adecuado (Rosales 1989). Asimismo, existen intereses industriales y energéticos por la biomasa de esta especie, especialmente para su trituración y obtención de pulpa de papel, que de manera general, indican que la demanda por biomasa forestal no maderable, es cada vez mayor (Bermúdez y Touza 2000). Así, según Noble y Herbert (1989), la biomasa sustentable depende de la disponibilidad de nutrientes, precipitaciones, capacidad de retención de agua del suelo y de las prácticas de manejo del sitio.

Por otro lado, las "Ecuaciones de Biomasa" nos permiten estimar la cantidad de biomasa que contiene un árbol en función de algunas de sus características, como su diámetro a la altura de pecho y altura de fuste. Estas ecuaciones se obtienen por medio de un análisis de regresión.

Con respecto a la biomasa aérea de plantaciones de E. globulus, se ha observado que a medida que se desarrolla la especie, la distribución porcentual de los distintos componentes de biomasa aérea (copa, ramas, fuste y corteza) registran un cambio natural con la edad, aumentando la proporción de madera, disminuyendo la de las ramas y hojas, mientras que la corteza después de algunos años se mantiene relativamente constante. El presente trabajo de investigación tiene como objetivo obtener la mejor ecuación para estimar la biomasa aérea de las plantaciones en linderos o cercos vivos de E. globulus, en el distrito de Huertas, provincia de Jauja, departamento de Junín.

\section{Materiales y Métodos}

\section{Zona de Estudio}

El estudio se llevó a cabo en los linderos plantados de Eucalyptus globulus en los terrenos de cultivos ubicados en el distrito de Huertas (al margen izquierdo del río Yacus), provincia de 
Jauja, departamento de Junín, Perú (Figura 1). Geográficamente el área distrital se localiza alrededor del punto de referencia: $11^{\circ} 46^{\prime}$ $07^{\prime \prime}$ latitud $\mathrm{S}$ y $75^{\circ} 28^{\prime} 37^{\prime \prime}$ longitud $\mathrm{O}$, con una superficie de $11,82 \mathrm{~km}^{2}$. El distrito de Huertas se encuentra ubicado a una altitud aproximada de $3365 \mathrm{msnm}$; posee un clima variado de acuerdo a las estaciones, entre templado, seco y lluvioso en los meses de diciembre a abril con una temperatura promedio de $14^{\circ}$ a $18^{\circ} \mathrm{C}$ y de mayo a noviembre le corresponde los meses más fríos del año, con una temperatura que llega a descender a valores bajo cero en junio y julio. Tiene una temperatura media anual de $10,9^{\circ} \mathrm{C}$ y una precipitación de $700 \mathrm{~mm}$. Por el sistema Thornthwaite, el clima es clasificado como B(i) $\mathrm{D}^{\prime} \mathrm{H} 3$, clima semi frígido, lluvioso, con lluvia deficiente en invierno, con humedad relativa calificada como húmeda (SENAMHI 2002).

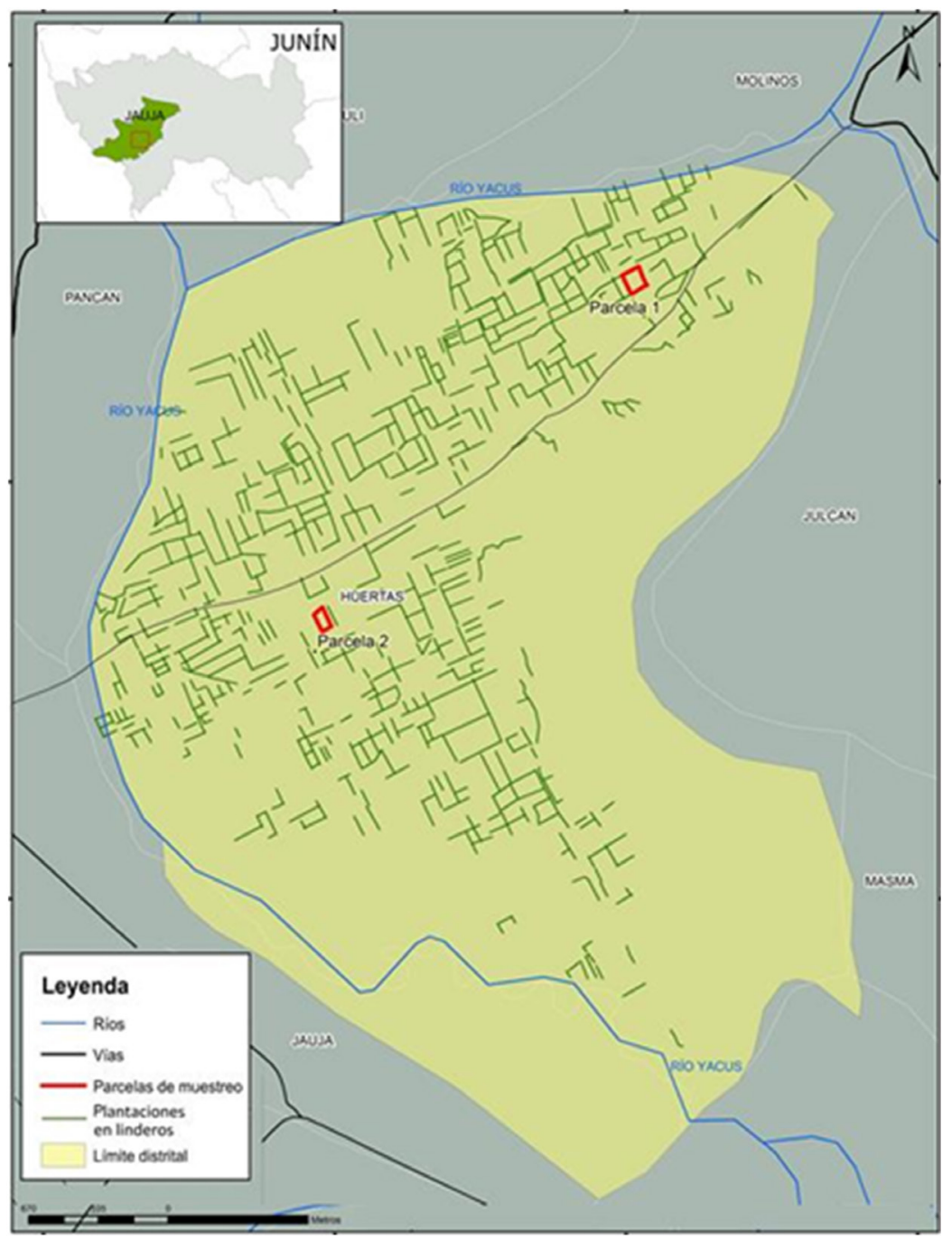

Figura 1. Ubicación del distrito y de cercos vivas. 


\section{Cálculo de la biomasa real}

\section{La muestra}

Un total de 40 árboles fueron seleccionados y marcados. Todos los individuos muestreados tenían un diámetro de altura del pecho (dap) mayor a $15 \mathrm{~cm}$. Esta muestra fue tomada en los límites de dos terrenos de cultivo con características representativas del distrito, cada uno con un límite perimétrico de cuatro lados constituidos por árboles de E. globulus que fueron plantados en diferentes años. De cada uno de los campos, se seleccionaron 20 árboles, que fueran representativos (en promedio y amplitud) de los diámetros, alturas y forma de copa de los límites de las chacras del distrito. Se debe mencionar que los límites seleccionados pertenecen a una muestra representativa del distrito de Huertas, con una intensidad de muestreo del $7 \%$ y un nivel de confianza del 95\%, resultados obtenidos por Llancari y Barrena (2016).

\section{Peso fresco}

Para la medición de la biomasa, se adaptó la metodología descrita en el "Manual de procedimientos de muestreo de biomasa forestal" (Schlegel et al. 2000). Primero, se midió el dap y las alturas de fuste y total de los 40 árboles en pie, con el uso de una cinta diamétrica y un hipsómetro. Luego, con ayuda de una moto-

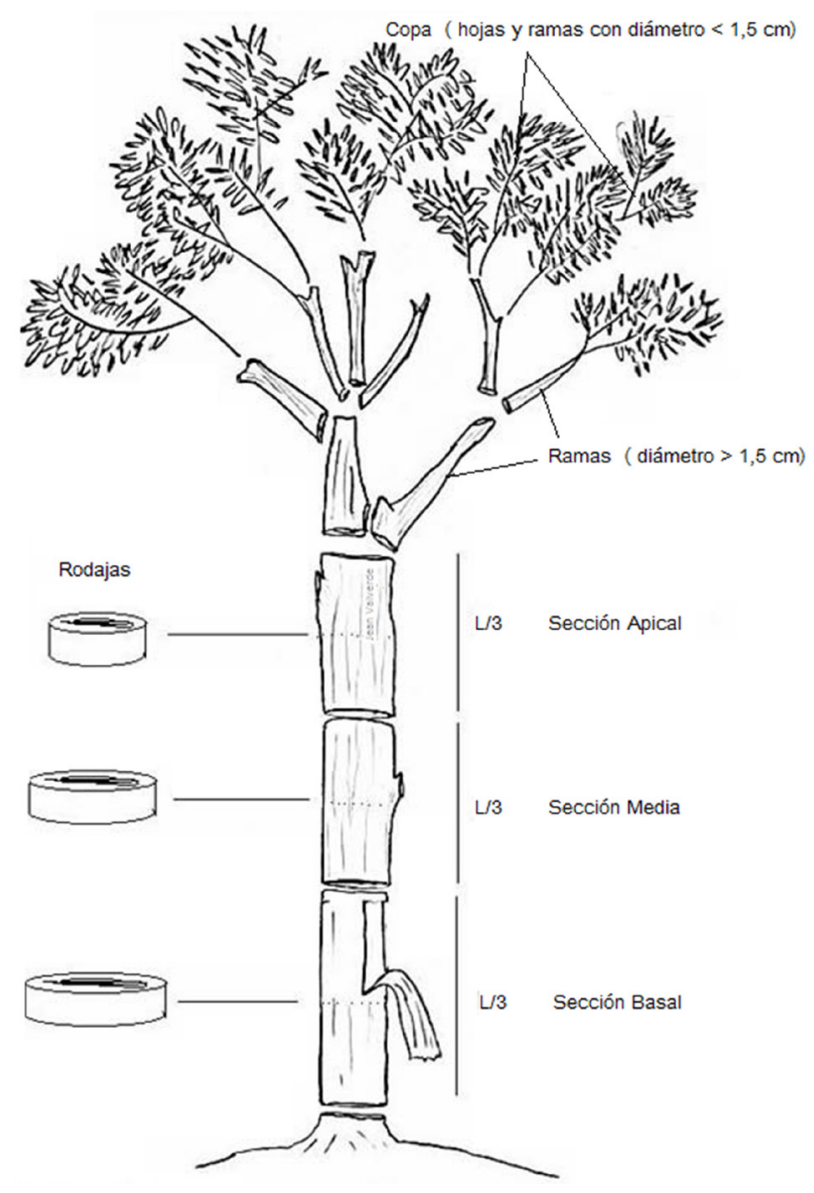

Figura 2. Componentes del árbol para su análisis y obtención de rodajas. 
sierra, cada árbol fue tumbado y su fuste fue dividido en tres secciones (baja, media y alta) de igual longitud. Asimismo, con una motosierra y machete, cada componente del árbol (fracciones de tronco, ramas y follaje) fue separado y dividido para su posterior pesaje (Figura 2). Para pesar cada componente, se montó un trípode con materiales de campo y se empleó una balanza romana de $0,5 \mathrm{~kg}$ de precisión. Así fueron pesadas la madera y la corteza de cada sección del fuste (bajo, medio y alto) por separado. Por su parte las ramas gruesas (de más de $1,5 \mathrm{~cm}$ de diámetro) fueron cortadas y atadas con una cuerda. Finalmente, el follaje formado por todas las hojas y ramitas terminales (iguales o menores a $1,5 \mathrm{~cm}$ de diámetro) se pesaron en grupos atados por una cuerda. Todas las cuerdas fueron pesadas previamente para descontar su peso, a la lectura de la balanza.

Luego del pesado, se extrajo una sección de $15 \mathrm{~cm}$ de largo de la parte media de cada sección del fuste de todos los árboles, de la cual se obtuvo dos muestras o probetas para la determinación del contenido de humedad y densidad de cada sección; también fueron seleccionadas, al azar, tres ramas y tres ramitas terminales (incluyendo sus hojas). Todas estas muestras fueron colocadas en bolsas de plástico herméticamente selladas.

\section{Cálculo de la biomasa}

Se determinó el contenido de humedad, para el fuste en los tres niveles del árbol, ramas gruesas, corteza y follaje, aplicando la formula recomendada en la Norma Técnica Peruana NTP 251.010 (INDECOPI 2004).

$$
\% \text { Humedad }=\frac{\text { Peso Fresco }- \text { Peso seco }}{\text { Peso seco }} \times 100
$$

La biomasa de cada componente del árbol se estimó utilizando el contenido de humedad y los pesos frescos obtenidos en el campo (Simbaña 2011).

$$
\text { Biomasa }=\frac{\text { Peso Fresco }}{1+C H \%}
$$

La biomasa total para cada árbol se calculó como la sumatoria de la biomasa de sus componentes.

\section{Ecuación para la estimación de biomasa aérea}

Antes de efectuar el análisis de regresión y de obtener las ecuaciones de biomasa, se evaluaron los datos para ver la existencia de "outliers" (Chatterjee y Price 1977), para ello se analizó gráficamente la relación biomasa y dap así como biomasa y la altura total. Los modelos utilizados para construir las ecuaciones para la estimación de biomasa aérea, tomados de Sim-

\begin{tabular}{|c|c|}
\hline Número & Modelos de Ecuaciones de Biomasa aérea \\
\hline 1 & $\mathrm{~B}=\mathrm{b}_{0}+\mathrm{b}_{1} \mathrm{dap}^{2}$ \\
\hline 2 & $\mathrm{~B}=\mathrm{b}_{0}+\mathrm{b}_{1} \mathrm{dap}^{2} \mathrm{~h}$ \\
\hline 3 & $\mathrm{~B}=\mathrm{b}_{0}+\mathrm{b}_{1}$ dap \\
\hline 4 & $\mathrm{~b}_{0}+\mathrm{b}_{1} \mathrm{dap}^{3}$ \\
\hline 5 & $\mathrm{~b}_{0}+\mathrm{b}_{1} \mathrm{dap}^{2} \mathrm{~h}^{3}$ \\
\hline 6 & $\mathrm{~b}_{0}+\mathrm{b}_{1} \mathrm{dap}^{3} \mathrm{~h}$ \\
\hline 7 & $\mathrm{~b}_{0}+\mathrm{b}_{1} \mathrm{dap}^{3} \mathrm{~h}^{3}$ \\
\hline 8 & $\mathrm{~b}_{0}+\mathrm{b}_{1} \mathrm{dap}^{2} \mathrm{~h}^{2}$ \\
\hline 9 & $\mathrm{~b}_{0}+\mathrm{b}_{1} \mathrm{dap}^{3} \mathrm{~h}^{2}$ \\
\hline 10 & $\mathrm{~b}_{0}+\mathrm{b}_{1} \mathrm{LN}(\mathrm{dap})$ \\
\hline 11 & $\mathrm{~b}_{0}+\mathrm{b}_{1}(\mathrm{dap})^{0,5}$ \\
\hline 12 & $\mathrm{~b}_{0}+\mathrm{b}_{1}(\mathrm{LN}(\mathrm{dap})) 1 / \mathrm{dap}$ \\
\hline 13 & $\mathrm{~B}=\mathrm{b}_{0}+\mathrm{b}_{1} \mathrm{dap}+\mathrm{b}_{2} \mathrm{dap}^{2} \mathrm{~h}$ \\
\hline 14 & $\mathrm{~B}=\quad \mathrm{b}_{0}+\mathrm{b}_{1} \mathrm{dap}+\mathrm{b}_{2} \mathrm{dap}^{2} \mathrm{~h}^{2}$ \\
\hline 15 & $\mathrm{~B}=\mathrm{b}_{0}+\mathrm{b}_{1} \mathrm{dap}^{2}+\mathrm{b}_{2} \mathrm{dap}^{2} \mathrm{~h}^{2}$ \\
\hline 16 & $\mathrm{~B}=\quad \mathrm{b}_{0}+\mathrm{b}_{1} \mathrm{dap}^{3}+\mathrm{b}_{2} \mathrm{dap}^{2} \mathrm{~h}^{2}$ \\
\hline 17 & $\mathrm{~B}=\quad \mathrm{b}_{0}+\mathrm{b}_{1} \mathrm{dap}^{3}+\mathrm{b}_{2} \mathrm{dap}^{2} \mathrm{~h}$ \\
\hline 18 & $\mathrm{~B}=\quad \mathrm{b}_{0}+\mathrm{b}_{1} \mathrm{dap}^{3}+\mathrm{b}_{2} \mathrm{dap}^{2} \mathrm{~h}^{3}$ \\
\hline 19 & $\mathrm{~B}=\quad \mathrm{b}_{0}+\mathrm{b}_{1} \mathrm{dap}^{3} \mathrm{~h}+\mathrm{b}_{2} \mathrm{dap}^{2} \mathrm{~h}^{3}$ \\
\hline 20 & $\mathrm{~B}=\quad \mathrm{b}_{0}+\mathrm{b}_{1} \mathrm{dap}^{2}+\mathrm{b}_{2} \mathrm{dap}^{3}$ \\
\hline 21 & $\mathrm{~B}=\quad \mathrm{b}_{0}+\mathrm{b}_{1}$ dap $+\mathrm{b}_{2} \mathrm{dap}^{3}$ \\
\hline 22 & $\operatorname{Ln} B=b_{0}+b_{1} \ln ($ dap $)$ \\
\hline 23 & $\operatorname{Ln} B=b_{0}+b_{1} \ln (h)$ \\
\hline 24 & $\operatorname{Ln} B=b_{0}+b_{1} \ln ($ dap $)+b_{2} \ln (h)$ \\
\hline 25 & $\operatorname{Ln} B=b_{0}+b_{1}[\ln (\text { dap })]^{2}+b_{2} \ln (h)$ \\
\hline 26 & $\operatorname{Ln} B=b_{0}+b_{1} \ln ($ dap $)+b_{2}[\ln (\text { dap })]^{2}$ \\
\hline 27 & $\operatorname{Ln} B=b_{0}+b_{1} \ln \left(\right.$ dap $\left.^{2} h\right)$ \\
\hline 28 & $\mathrm{Ln} B=\mathrm{b}_{0}+\mathrm{b}_{1} \ln \left(\right.$ dap $\left.^{2} \mathrm{~h}\right)+\mathrm{b}_{2} \ln (\mathrm{h})$ \\
\hline
\end{tabular}
baña (2011), se muestran en el Cuadro 1.

Cuadro 1. Modelos de ecuación de regresión. 


\section{Análisis de regresión basado en el Método de los Mínimos Cuadrados}

Se obtuvieron ecuaciones de biomasa luego de aplicar el Método de los Mínimos Cuadrados a un nivel de significación del 0,05. Se rechazaron las ecuaciones que no cumplieron las pruebas propias del análisis de regresión: (i) para tener ecuación con un buen ajuste, el coeficiente de determinación $\left(\mathrm{R}^{2}\right)$ de cada ecuación debe ser mayor a 0,8 para modelos lineales y 0,75 para los modelos logarítmicos (Alvarado y Obagi 2008), (ii) para conocer si la regresión lineal es significativa se utilizó Fisher, aceptando o rechazando la ecuación según los criterios mostrados en el Cuadro 2, y (iii) para conocer si cada una de las variables independientes es significativa en la ecuación ( $\mathrm{si}$ bi $\neq 0$ ), se utilizó la prueba de significancia t-student, aceptando o rechazando la ecuación según los criterios mostrados en el Cuadro 3.

\section{Análisis de residuos}

Para cada ecuación se analizó si la distribución de los residuos era homocedástico (Chatterjee y Price 1977, Montgomery 2004, Vilar 2006), en caso contrario, el modelo de la ecuación fue ponderado posteriormente; también fueron observados distribuciones no lineales y datos atípicos.

\section{Regresión ponderada (mínimos cuadrados ponderados)}

Los modelos de las ecuaciones que presentaron heterocedasticidad en el Análisis de los residuos, fueron ponderados (Barrena 1988) varias veces por un factor " $K_{i}$ ":

$$
\frac{\text { Biomasa aérea }}{K}=\frac{b_{0}}{K}+\frac{\mathrm{X}_{\mathrm{i}}}{K}
$$

Para un $\mathrm{K}_{\mathrm{i}}=\left(\mathrm{X}_{\mathrm{i}}\right) \mathrm{u}$. donde $\mathrm{u}=0,2 ; 0,3 ; 0,4 ; \ldots ; \mathrm{u}_{\mathrm{i}}$

Se emplearon distintos valores de $\mathrm{u}$, de manera progresiva hasta lograr la homogeneidad de variancias de los errores. A cada modelo ponderado se le realizó el análisis de regresión de igual manera que a las ecuaciones sin ponderar, aceptando y rechazando a las ecuaciones de biomasa ponderadas si cumplían o no con los criterios ya mostrados.

\section{Selección de la mejor ecuación}

Para determinar cuál de las ecuaciones estimaba mejor la biomasa aérea, a las ecuaciones ponderadas aceptadas y que mostraron homocedasticidad en el análisis de residuos, se les calculó el Índice de Furnival (Furnival 1961) para ecuaciones ponderadas (Barrena et al. 1986).

$$
I F=\sqrt{S} \times \operatorname{anti} \log \left(\frac{1}{n} \times \sum \log p\right)
$$

Donde:

IF = Índice de Furnival

$$
\mathrm{S}=\text { Cuadrado Medio del Error }
$$

$\mathrm{n}=$ número de árboles de la muestra

$\mathrm{p}=$ valor numérico del factor $\mathrm{K}_{\mathrm{i}}$

Se seleccionó la ecuación ponderada que presentó el menor valor del IF. Finalmente, la ecuación de biomasa ponderada seleccionada, fue transformada, de acuerdo con Valverde (2017), multiplicándola por el factor K y así obtener la ecuación que estima mejor la biomasa aérea de los árboles de E. globulus, de esta manera el ejemplo de modelo ponderado (a), toma la forma siguiente:

$$
\text { Biomasa aérea }=b_{0}+b_{1} X
$$

\begin{tabular}{|l|l|}
\hline \multicolumn{1}{|c|}{ Condición } & \multicolumn{1}{c|}{ Decisión } \\
\hline $\begin{array}{l}\text { F calculado }>\text { F tabular } \\
(p \text {-valor }>0,05)\end{array}$ & $\begin{array}{l}\text { El modelo lineal es signifi- } \\
\text { cativo, la ecuación se acepta }\end{array}$ \\
\hline $\begin{array}{l}\text { F calculado }<\text { F tabular } \\
(p \text {-valor }<0,05)\end{array}$ & $\begin{array}{l}\text { El modelo es constante, no } \\
\text { significativo, la ecuación se } \\
\text { rechaza }\end{array}$ \\
\hline
\end{tabular}

Cuadro 2. Criterio de decisión para la prueba de significancia Fisher.

\begin{tabular}{|l|l|}
\hline \multicolumn{1}{|c|}{ Condición } & \multicolumn{1}{|c|}{ Decisión } \\
\hline $\begin{array}{l}\mathrm{t} \text { calculado }>\mathrm{t} \text { tabular } \\
(p \text {-valor }>0,05)\end{array}$ & $\begin{array}{l}\text { La variable } \mathrm{x}_{\mathrm{i}} \text { es signifi- } \\
\text { cativa en } \mathrm{Y} \text { (se acepta la } \\
\text { ecuación) }\end{array}$ \\
\hline $\begin{array}{l}\mathrm{t} \text { calculado }<\mathrm{t} \text { tabular } \\
(p \text {-valor }<0,05)\end{array}$ & $\begin{array}{l}\text { La variable } \mathrm{x}_{\mathrm{i}} \text { no tiene un } \\
\text { efecto significativo sobre } \mathrm{Y} \\
\text { (se rechaza la ecuación) }\end{array}$ \\
\hline
\end{tabular}

Cuadro 3. Criterio de decisión para la prueba de significancia t-student. 


\section{Resultados}

\section{La muestra}

El promedio de la altura total, la altura al punto de copa y el dap de los árboles muestreados fueron: $18,87 \mathrm{~m} ; 15,05 \mathrm{~m}$ y $20,05 \mathrm{~cm}$ respectivamente. Los valores máximos y mínimos de la altura total, altura al punto de copa y dap, fueron: 23,75 y 14 m; 11,75 y 18 m; 16,71 y $36,76 \mathrm{~cm}$ respectivamente.

\section{Contenido de humedad $(\% \mathrm{CH})$}

Se encontró que la corteza tiene el mayor promedio de contenido de humedad, seguido por las hojas y finalmente las ramas, 127,39; 92,61 y 83,39 por ciento respectivamente (Cuadro 4). La madera muestra valores decrecientes desde la base hacia el ápice del árbol, los valores son 114,03 por ciento para la sección basal, 104,21 por ciento para la sección media y 87,22 por ciento para la sección apical del fuste, como se grafica en la Figura 3.

\section{Biomasa}

En el Cuadro 5 se observa que el fuste posee el 69,03 por ciento de la biomasa total, seguida de la copa (incluye hojas y ramitas terminales) con 15,39 por ciento y la corteza, ramas y tocón con 8,02 por ciento, 6,40 por ciento y 1,14 por ciento respectivamente. La biomasa promedio (en kilogramos) por cada componente del árbol y sección del fuste se grafica en la Figura 4, en donde se aprecia que la copa contribuye más en la biomasa total que la corteza, ramas, tocón y la sección apical del fuste.

\section{Análisis de regresión}

El árbol 30 fue retirado de la base de datos, porque se le consideró un "outlier" como lo demuestran las Figuras 5 y 6 . Entonces, las ecuaciones de biomasa para el presente estudio fueron elaboradas con 39 árboles.

Las ecuaciones de los modelos 1, 3, 4, 10, 11, 12 y 26 (Cuadro 1) presentaron valores de R2 menores a los umbrales establecidos $(0,8$

\begin{tabular}{crrrrrr}
\hline \multicolumn{7}{c}{ Contenido de humedad (\%) } \\
\hline & \multicolumn{7}{c}{ Fuste } & \multicolumn{1}{c}{ Ramas } & Corteza & Hoja \\
\cline { 2 - 5 } & \multicolumn{1}{c}{ Basal } & \multicolumn{1}{c}{ Media } & \multicolumn{1}{c}{ Apical } & & & \\
\hline Promedio & 114,03 & 104,21 & 87,22 & 83,39 & 127,39 & 92,61 \\
Máximo & 147,79 & 143,52 & 125,05 & 101,89 & 154,27 & 141,67 \\
Mínimo & 75,05 & 68,16 & 70,32 & 69,67 & 90,63 & 58,88 \\
CV\% & 15,09 & 16,85 & 13,65 & 10,56 & 11,53 & 19,15 \\
\hline
\end{tabular}

Cuadro 4. Contenido de humedad $(\% \mathrm{CH})$ por cada componente del árbol.

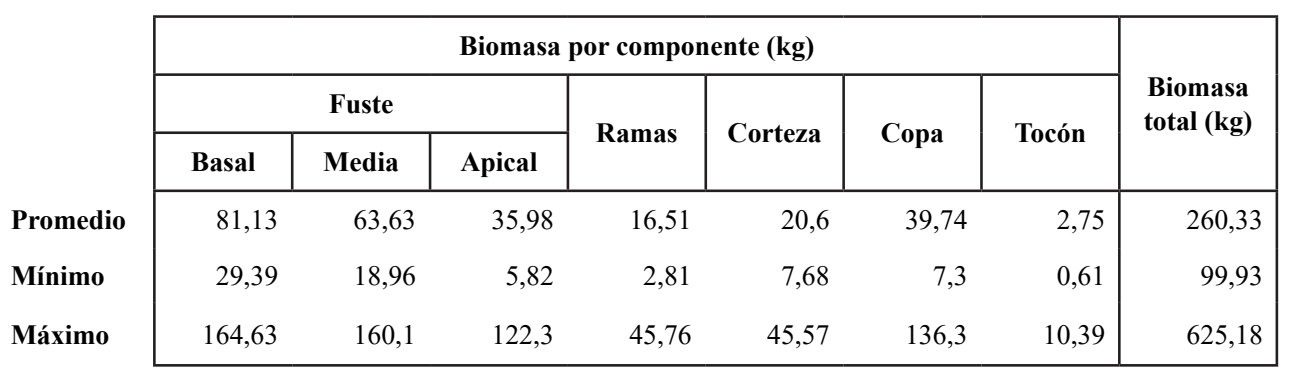

Cuadro 5. Biomasa por componente del árbol. 
para los modelos lineales y 0,75 para los logarítmicos). Eso quiere decir que solo pasaron al siguiente paso, 21 ecuaciones. Asimismo, se apreció que aquellos modelos lineales que solo presentaron el dap como única variable independientes fueron rechazados en este primer análisis, al presentar los valores de $\mathrm{R}^{2}$ más bajos. Caso contrario, todos los modelos que incluyen la altura total (h) en sus variables fueron aceptados.

En cuanto la prueba de significancia Fisher, todas las ecuaciones fueron aceptadas ya que sus valores de $p$-valor fueron menores a 0,05. Todas las ecuaciones pasaron a la siguiente etapa del análisis. Por otro lado, como se aprecia en el Cuadro 6, no todas las ecuaciones demostraron, con la prueba de $t$, que algún $b_{i}$ fuera diferente a 0 , estas ecuaciones fueron rechaza- das (ecuaciones $13,14,15,16$ y 17 ) y no pasaron a la siguiente etapa del análisis. Solo 12 ecuaciones fueron consideradas para el siguiente análisis $(2,5,6,7,8,9,18,19$ y las logarítmicas $24,25,27$ y 28$)$.

El Análisis gráfico de los residuales indico que todas las ecuaciones $(2,5,6,7,8,9,18$, 19 y las logarítmicas $24,25,27$ y 28 ) presentaron heterocedasticidad, es decir los errores (o residuos), no presentaron una variancia constante, lo cual se aprecia con los ejemplos en la Figura 7. Así las ecuaciones 2, 5, 6, 7, 8, 9, 18, 19 fueron ponderadas para realizar el Análisis de regresión ponderado. Las ecuaciones logarítmicas $(24,25,27$ y 28$)$, que también presentaban heterocedasticidad, no fueron ponderados pues se consideró que ya habían sido transformadas.

Prueba de Significancia (t-student)

\begin{tabular}{|c|c|c|c|c|c|c|c|}
\hline Modelo & $t b_{1}$ & t -tabular & $p-v a l o r$ & $t b_{2}$ & t -tabular & $p$ - valor & Calificación \\
\hline 2 & 14,6485 & 1,686 & $5,58 \mathrm{E}-17$ & & & & Aceptado \\
\hline 5 & 13,712 & 1,686 & $4,44 \mathrm{E}-16$ & & & & Aceptado \\
\hline 6 & 14,4843 & 1,686 & $7,98 \mathrm{E}-17$ & & & & Aceptado \\
\hline 7 & 14,2376 & 1,686 & $1,37 \mathrm{E}-16$ & & & & Aceptado \\
\hline 8 & 14,654 & 1,686 & $5,52 \mathrm{E}-17$ & & & & Aceptado \\
\hline 9 & 14,9961 & 1,686 & $2,65 \mathrm{E}-17$ & & & & Aceptado \\
\hline 13 & $-1,1083$ & 1,686 & 0,2751 & 5,1358 & 1,686 & $9,91 \mathrm{E}-06$ & Rechazado \\
\hline 14 & 0,8645 & 1,686 & 0,3931 & 5,0607 & 1,686 & $1,25 \mathrm{E}-05$ & Rechazado \\
\hline 15 & 1,1837 & 1,686 & 0,2443 & 4,3066 & 1,686 & 0,0001 & Rechazado \\
\hline 16 & 1,4548 & 1,686 & 0,1544 & 4,1471 & 1,686 & 0,0002 & Rechazado \\
\hline 17 & $-0,1167$ & 1,686 & 0,9077 & 3,7724 & 1,686 & 0,0006 & Rechazado \\
\hline 18 & 2,6598 & 1,686 & 0,0116 & 4,2115 & 1,686 & 0,0002 & Aceptado \\
\hline 19 & 2,6753 & 1,686 & 0,0112 & 1,8302 & 1,686 & 0,0755 & Aceptado \\
\hline 24 & 6,51 & 1,686 & $1,45 \mathrm{E}-07$ & 2,4169 & 1,686 & 0,0208 & Aceptado \\
\hline 25 & 6,5993 & 1,686 & $1,11 \mathrm{E}-07$ & 2,4196 & 1,686 & 0,0207 & Aceptado \\
\hline 27 & $-2,8057$ & 1,686 & 0,008 & 10,6871 & 1,686 & $7,27 \mathrm{E}-13$ & Aceptado \\
\hline 28 & 6,51 & 1,686 & $1,45 \mathrm{E}-07$ & $-0,3017$ & 1,686 & 0,7646 & Aceptado \\
\hline
\end{tabular}

Cuadro 6. Resultados de la prueba t-student. 


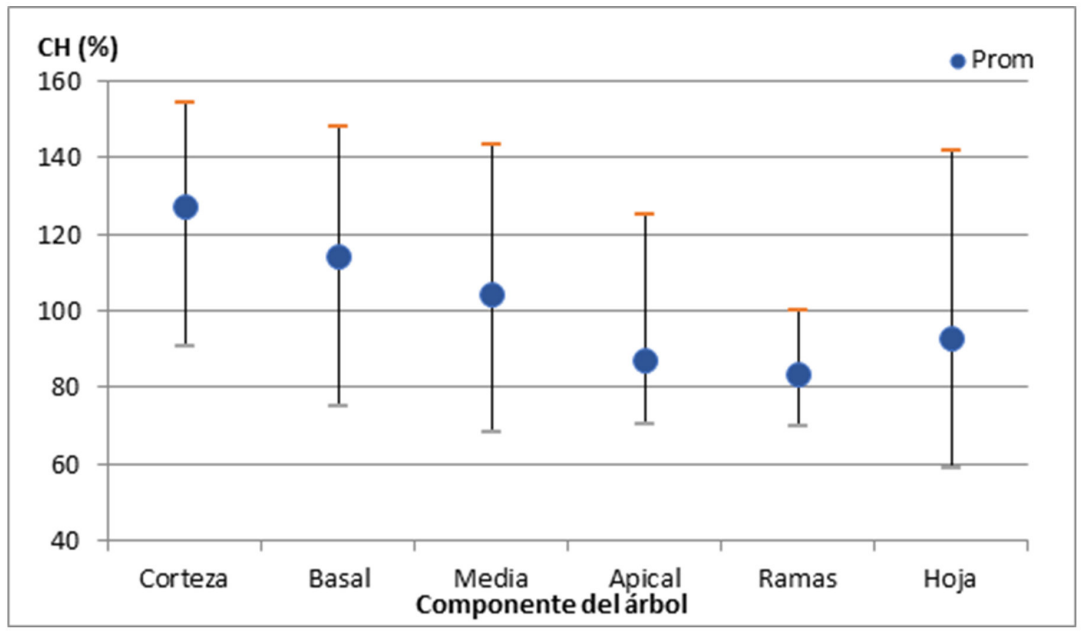

Figura 3. Valores promedio, máximo, mínimo del contenido de humedad por componente del árbol.

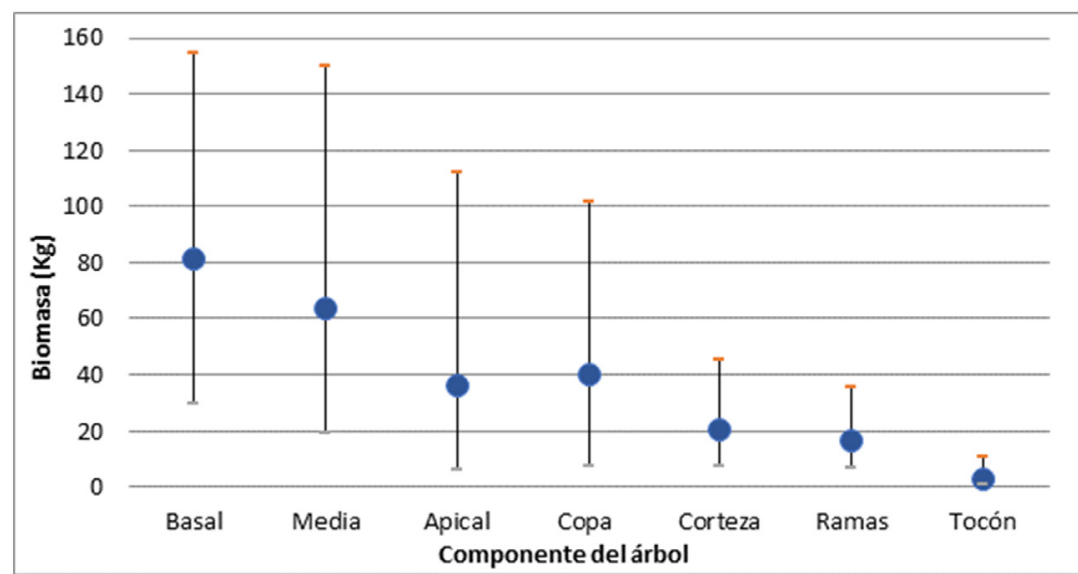

Figura 4. Valores promedio, máximo y mínimo de biomasa promedio por componente del árbol.

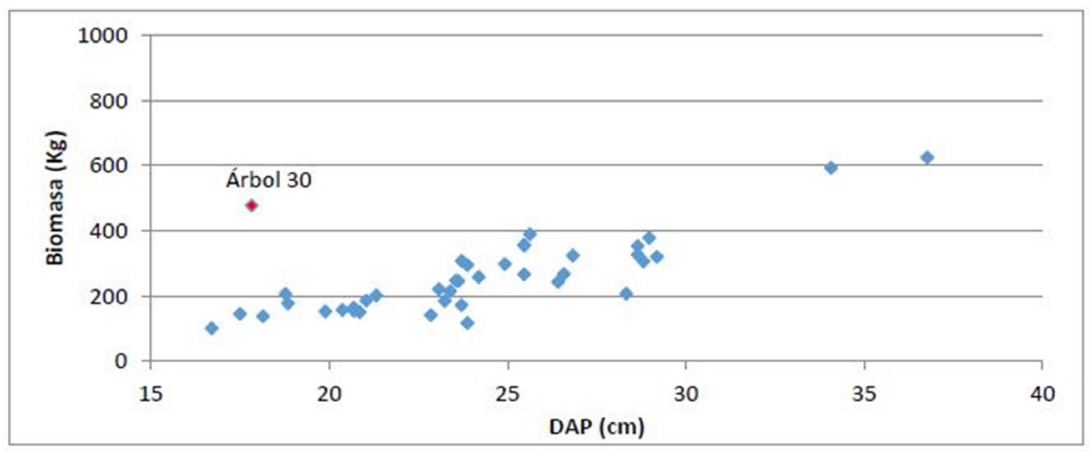

Figura 5. Relación de la Biomasa total y el dap (40 árboles). 


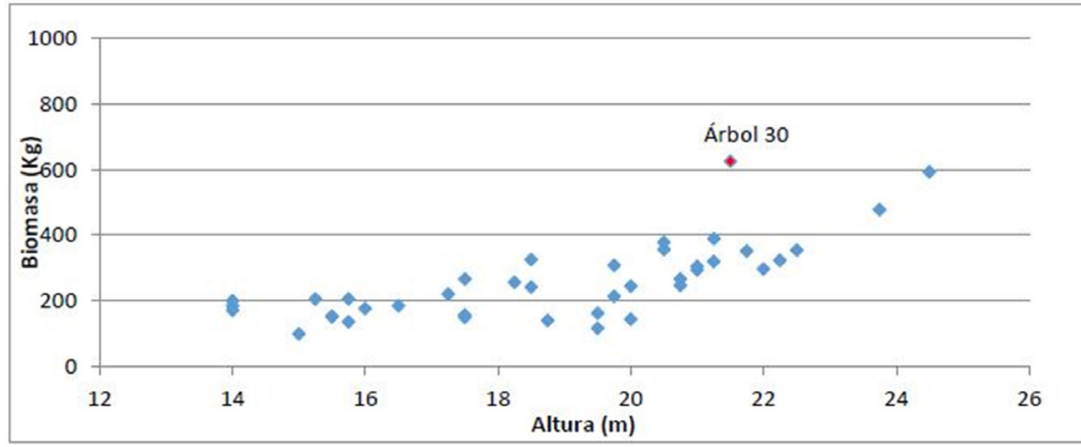

Figura 6. Relación de la Biomasa total y la altura total (40 árboles).

\section{Regresión ponderada}

Se elaboraron 48 ecuaciones de regresión ponderadas a partir de las 8 ecuaciones mencionadas en el párrafo anterior. Las únicas ecuaciones que logaron homogenizar la varianza de sus errores (o residuos) fueron las 2, 5, 6 y 8 (Figura 8) usando diferentes factores de ponderación: $\left(\text { dap }^{2} h\right)^{0,9},($ daph $),\left(\text { dap }^{3} h\right)^{0,5}$ y $\left(\text { dap }^{2} h\right)^{0,7}$ respectivamente. Estas cuatro ecuaciones no fueron rechazadas luego de analizar los resultados con el $\mathrm{R}^{2}$, la prueba de $\mathrm{F}$ y la prueba de $\mathrm{t}$.

\section{Selección de la mejor ecuación}

En el Cuadro 7 se presentan los valores del Índice de Furnival (IF) para las 4 ecuaciones ponderadas seleccionadas, se aprecia que la ecuación 8 es la que tiene el menor IF que luego de ser transformada (Valverde 2017) es la ecuación que mejor estima la biomasa aérea árboles de E. globulus:

Biomasa aérea $=90,45674738+0,00710053$ dap $^{2} h^{2}$

\begin{tabular}{|l|c|c|}
\hline \multicolumn{2}{|c|}{ Ecuaciones ponderadas } & Índice Furnival \\
\hline Ecuación 2 & $\left(\right.$ Biomasa aérea $=b_{0}+b_{1}$ dap $\left.^{2} h\right) /\left(\text { dap }^{2} h\right)^{0,9}$ & 0,00016881 \\
\hline Ecuación 5 & $\left(\right.$ Biomasa aérea $=b_{0}+b_{1}$ dap $\left.^{2} h^{3}\right) /($ dap $h)$ & 0,00499173 \\
\hline Ecuación 6 & $\left(\right.$ Biomasa aérea $=b_{0}+b_{1}$ dap $\left.^{3} h\right) /\left(\text { dap }^{3} h\right)^{0,5}$ & 0,00398338 \\
\hline Ecuación 8 & $\left(\right.$ Biomasa aérea $=b_{0}+b_{1}$ dap $\left.^{2} h^{2}\right) /\left(\text { dap }^{2} h\right)^{0,7}$ & 0,00012922 \\
\hline
\end{tabular}

Cuadro 7. Valores del Îndice de Furnival, selección de la mejor ecuación.

\section{Discusión}

\section{Contenido de humedad $(\% \mathrm{CH})$}

La relación del contenido de humedad encontrada, concuerda con los resultados de Simbaña (2011), cuyos promedios del contenido de humedad para corteza y hojas fueron $123,59 \mathrm{y}$ 105,39 por ciento, respectivamente; así mismo, 99,51 por ciento para la madera basal, 90,60 por ciento para la madera de la sección media y 89,60 por ciento para la madera de la sección alta; hay que mencionar que ambos estudios se desarrollaron en los Andes del Centro (Josse et al. 2009).

Se puede apreciar en el Cuadro 4 que el contenido de humedad para las ramas posee una menor variabilidad que los otros componentes. Este comportamiento puede deberse a que se tienen unidades muéstrales con características semejantes; al definir un diámetro mínimo para las ramas se disminuye la variabilidad de este componente. Así mismo, una de las 
características de la especie es tener un mayor contenido de humedad en su madera con menor densidad. Sin embargo esta humedad está conformada principalmente de agua libre fácilmente eliminable, lo cual aumenta su variabilidad. Por lo tanto, hay una menor variabilidad en madera de mayor densidad; esto se debe porque contiene menor proporción de agua libre y mayor agua higroscópica y de agua de constitución que son difícil de eliminar por el medio ambiente. La hoja muestra la mayor variabilidad en los valores de contenido de humedad, al mostrar máximos y mínimos bastante alejados del promedio. La variabilidad de la hoja puede deberse a la naturaleza propia de la copa, de ganar y perder humedad con mayor o menor facilidad dependiendo de las condiciones climáticas en las que se encuentre; es decir, un día previo de lluvia puede saturar las hojas con mayor facilidad que a las ramas y el fuste del árbol, y un día soleado puede disminuir el contenido de humedad con la misma facilidad.

\section{Biomasa}

La relación entre la biomasa de los componentes, coincide con las obtenidas por Simbaña (2011), Ribeiro et al. (2015), Âlvarez et al. (2005) y Herrera (1977), cuyos resultados para la misma especie muestran al fuste como mayor contribuyente en la biomasa aérea total; sin embargo, estos autores atribuyen un mayor
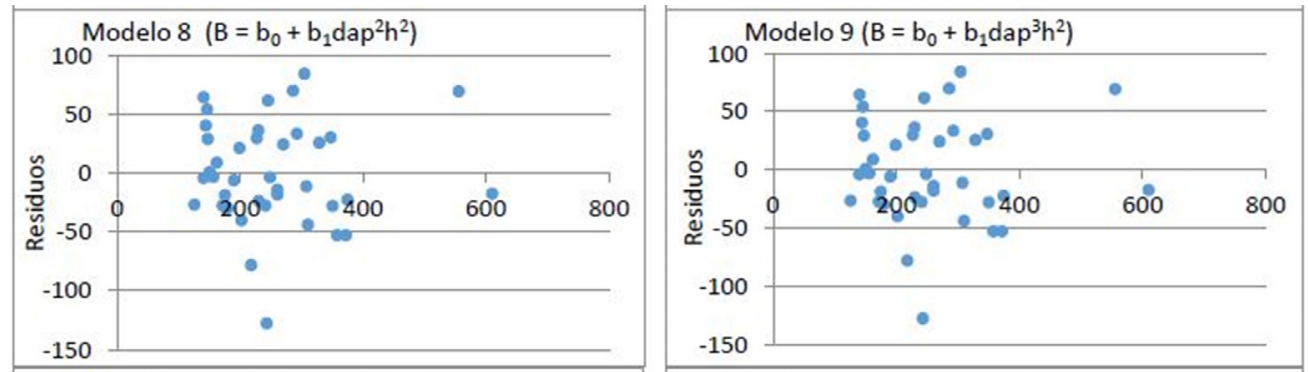

Figura 7. Ejemplos de heterocedasticidad. Ecuaciones 8 y 9.
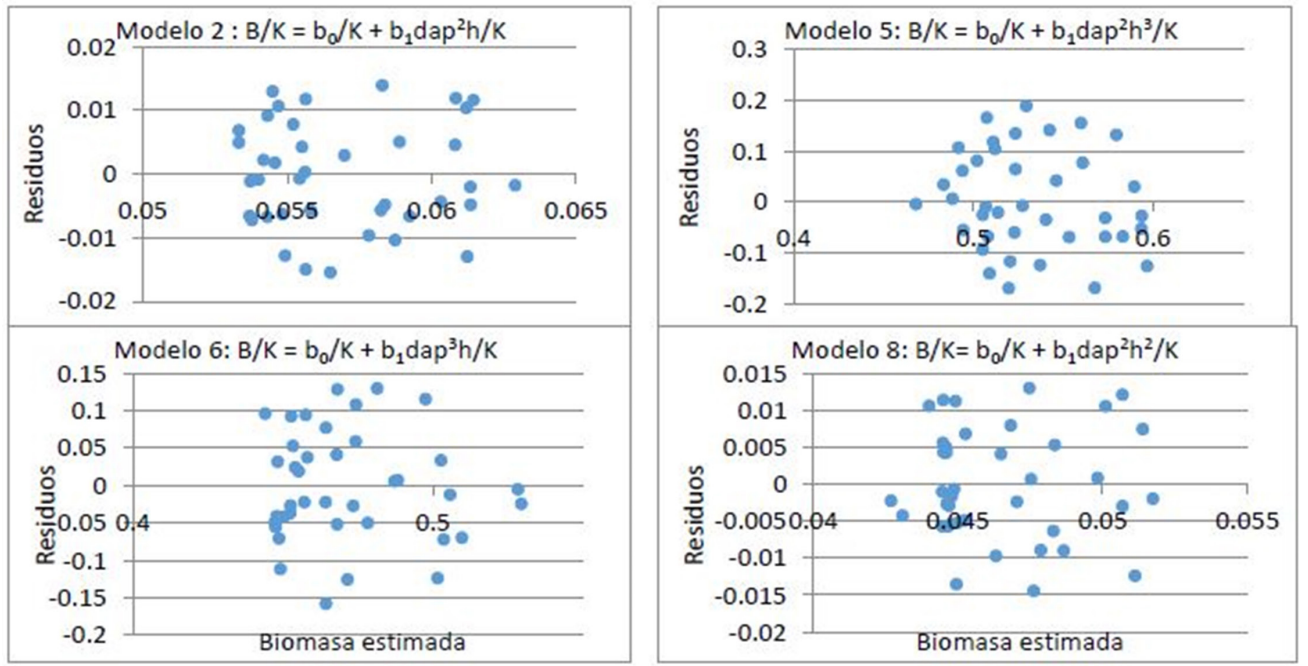

Figura 8. Ejemplos de homocedasticidad. Ecuaciones 2, 5, 6 y 8 ponderadas. 
porcentaje a la corteza sobre las hojas. Por otro lado, el hecho que la copa contribuya más que la corteza, ramas, tocón y la sección apical del fuste, se puede deber a que se trata de árboles que no han recibido ningún tratamiento silvicultural, permitiendo el crecimiento frondoso de sus copas.

\section{Análisis de regresión}

Se puede apreciar que las ecuaciones que solo presentan al dap, sea de manera lineal, al cuadrado, al cubo o en Ln como única variable independientes fueron rechazados en este primer análisis, en cambio las ecuaciones que, además del dap incluyen la altura total (h) fueron aceptados. Lo cual denota la importancia de tener más de una variable para estimar esta biomasa.

De los resultados de la prueba de Fisher, se infiere que el modelo lineal es significativo, es decir que la variable dependiente (biomasa) depende de las variables dasométricas independientes (dap y altura).

Los resultados de la prueba de $t$ indican que no todas las variables independientes influyen significativamente en la biomasa aérea. Por ejemplo, la ecuación 14 tiene como variables independientes dap y dap ${ }^{2} h^{2}$, esta última variable, es la única variable independiente en la ecuación 8 que ha demostrado tener un buen ajuste, que existe una dependencia entre ella y la biomasa, pero la otra variable dependiente (dap) está considerada en otra ecuación (la 3), ecuación que ha sido eliminada por no presentar un buen ajuste; es decir que en esta etapa, también hay que considerar la combinación de variables independientes para explicar la eficiencia de una ecuación para estimar biomasa.

Para corregir la heterocedasticidad, y así cumplir con una de la hipótesis de base de los mínimos cuadrados, es necesario ponderar las ecuaciones (Chatterjee y Price 1977, Montgomery et al. 2006, Vilar 2006). Barrena (1988) confirma que la heterocedasticidad está presente en la relación del volumen con el dap y h, por lo que también es probable encontrarla en la estimación de la biomasa como en este caso.

\section{Regresión ponderada}

Un aspecto a resaltar de las 4 ecuaciones ponderadas seleccionadas es que, a diferencia de lo encontrado por Barrena (1998) en la estimación del volumen, los valores del $\mathrm{R}^{2}$ de las ecuaciones ponderadas son mayores que los valores de $\mathrm{R}^{2}$ de las ecuaciones originales correspondientes. Esto puede deberse a que, si el factor de ponderación contiene una variable diferente a la variable independiente, se puede decir que la ecuación ponderada tiene una variable más que la original, y según, Barrena et al. (1986) y Rodríguez (2005), el R² aumenta al incrementar el número de variables independientes.

La ecuación dap ${ }^{2} h$ es considerada en la teoría la mejor combinación de variables para explicar el volumen del fuste, sin embargo, en el presente estudio se demostró que dap ${ }^{2} h^{2}$ explica mejor la biomasa del árbol en su conjunto (fuste y copa). Por lo tanto, esta última combinación de variables mostró un mejor ajuste para la estimación de biomasa aérea de las plantaciones de E. globulus en la zona de estudio.

Los resultados encontrados pueden ser la base para estimar la producción de productos maderables como no maderables (Moreno 2015) de esta especie en la región; así como para estimar su oferta energética (Barrena et al. 2010); de igual manera, podría ser usados en los cálculos en el sector Uso del Suelo, Cambio de Uso del Suelo y Silvicultura (USCUSS) dentro de los Inventarios Nacionales de Gases de Efecto Invernadero (INGEI) del Perú (MINAM 2016).

\section{Conclusiones}

La ecuación que mejor estima la biomasa aérea de las plantaciones en linderos o cercos vivos de E. globulus, en el distrito de Huertas, provincia de Jauja, departamento de Junín es:

Biomasa aérea $=90,45674738+0,00710053$ dap $^{2} h^{2}$

Además, el contenido de humedad en los árboles de E. globulus disminuye de la parte basal a la zona apical del árbol. 


\section{Agradecimientos}

Al Círculo de Investigación y Conservación en el Ámbito Forestal (CICAF) de la Facultad de Ciencias Forestales de la UNALM y a sus miembros: Efraín Suárez, Erick Suárez, Jorge Luis Cornejo, Pablo Chenchín, Melissa Huamantinco, Yuri Llancari, Paola Monzón, José Carlos Quispe, Manuel Gerardo, Luis Enrique Titto y Alex Machacuay, por su apoyo en la realización del trabajo de campo.

\section{Bibliografía}

Alvarado, J; Obagi, J. 2008. Fundamentos de inferencia estadística. Pontificia Universidad Javeriana, Bogotá. 361 p.

Álvarez, JG; Balboa, MA; Merino, A; Rodriguez, R. 2005. Estimación de la biomasa arbórea de Eucalyptus globulus y Pinus pinaster en Galicia. Recursos Rurais 1(1): 21-30.

Barrena, V; Gianella, J; García, H.; Flores, N.; Rubín De Celis, E.; Ocaña, J.; Guillén, R. 2010. Análisis de residuos biomásicos leñosos y de residuos para uso combustible. In Félix, E; Rosell, C (eds). Bionergía y seguridad alimentaria. El análisis de BEFS para el Perú. Compendio técnico. Vol 1. Resultados y conclusiones. FAO Documento de trabajo: Manejo del Ambiente y los Recursos Naturales; Bionergía. $\mathrm{N}^{\circ}$ 38. pp. 71-95.

Barrena, V. 1988. La regresión ponderada en la elaboración de las ecuaciones de volumen. Revista Forestal del Perú 15(2): 21- 28.

Barrena, V; Dancé, J; Sáenz, D. 1986. Metodología para la selección de ecuaciones de volumen. Revista Forestal del Perú. 13(2): 3-12.

Bermúdez, J; Touza, M. 2000. Las cifras del Tercer Inventario Forestal de Galicia y su incidencia en la industria de la transformación de la madera Revista CIS-Madera 4. 6-24 p.

Chatterjee, S; Price, B. 1977. Regression Analysis by Example. Wiley, J. \& Sons, INC., publication. $230 \mathrm{p}$.

Furnival, G. 1961. An Index for comparing equations used in constructing volume tables. Forest Science. 7(4): 337 - 341.
Herrera, A. 1977. La madera como materia prima natural. Ingeniería Química. 42 p.

INDECOPI (Instituto Nacional de Defensa de la Competencia y de la Protección de la Propiedad Intelectual). 2004. Norma Técnica Peruana 251.010 Lima PE. 24 p.

Josse, C; Cuesta, F; Navarro, G; Barrena, V; Cabrera, E; Chacón-Moreno, E; Ferreira, W; Peralvo, M; Saito, J; Tovar, A. 2009. Ecosistemas de los Andes del Norte y Centro. Bolivia, Colombia, Ecuador, Perú y Venezuela. Secretaría General de la Comunidad Andina, Programa Regional ECOBONA-Intercooperation, CONDESAN-Proyecto Páramo Andino, Programa BioAndes, EcoCiencia, NatureServe, IAvH, LTA-UNALM, ICAE-ULA, CDCUNALM, RUMBOL SRL. Lima. 98 p.

Llancari, Y; Barrena, V. 2016. Desarrollo de un diseño de inventario para linderos de eucalipto en parcelas agrícolas en el distrito de Huertas, provincia de Jauja, region Junín. Informe de investigación. UNALM. Lima, 12 p.

MINAG (Ministerio de Agricultura y Riego). 2013. Perú Forestal en números. Año 2012. Dirección General Forestal y de Fauna Silvestre. Lima. 203 p + anexos.

MINAM (Ministerio del Ambient). 2016. Inventario nacional de efectos invernadero (INGEI) 2012, 2005 y actualización de INGEI 2010 y 2000. 336 p. Consultado 28 ene 2019. Disponible en https://www.gob.pe/institucion/ minam/informes-publicaciones/2621-inventario-nacional-de-gases-de-efecto-invernadero-ingei-2012-2005-y-actualizacion-de-ingei-2010-y-2000

Montgomery, D. 2004. Diseño y análisis de experimentos. Segunda edición. Universidad estatal de Arizona. 700 p.

Moreno L. 2015. Manejo e industrialización del Eucalipto (Eucalyptus globulus). Tesis Ing. Agroind. UNT. 36 p.

Noble, A; Herbert M. 1989. Estimated nutrients removal in a short rotation Eucalyptus grandis crop on a fernwood soil. I.C.F.R. Annual Report 1989: 139-150. 
Ribeiro, S. C; Soares, C. P. B; Fehrmann, L; Jacovine, L. A. G; von Gadow, K. 2015. Aboveground and belowground biomass and carbon estimates for clonal Eucalyptus trees in Southeast Brazil. Revista Árvore 9(2): 353-363.

Rodríguez, M. 2005. Errores frecuentes en la interpretación del coeficiente de determinación lineal. Anuario jurídico y económico escurialense (38): 315-331.

Rosales, UD. 1989. La utilización de Eucaliptus globulus. Dirección General de Fomento Forestal y de Fauna. Instituto Nacional Forestal y de Fauna. Madrid, España. 26 p.

Schlegel, B; Gayoso, J; Guerra, J. 2000. Manual de procedimientos muestreos de biomasa forestal. Proyecto Medición de la capacidad de captura de carbono en bosques de Chile y promoción en el mercado mundial. FONDEFUACh-INFOR. 20 p.

SENAMHI (Servicio Nacional de Meteorología e Hidrología) 2002. Mapa de Clasificación Climática por el Método de Werren Thornthwaite. Consultado Abril 2016. Disponible en https://debconsulting.weebly.com/ peruacute-clasif-climat-senamhi---wt.html

Simbaña, N. 2011. Estimación de la captura de carbono en plantaciones de Eucalyptus globulus Labill. Tesis Mg, Sc, EPG-UNALM. PE. 120 p.

Valverde, J. 2017. Determinación de la ecuación de biomasa aérea de Eucalyptus globulus Labill en plantaciones en cercos vicos, distrito de Huertas, Junín. Tesis Ing. For. FCF-UNALM. $86 \mathrm{p}+$ anexos.

Vilar, J. 2006. Modelos estadísticos aplicados. Segunda edición. Universidad de Santiago de Compostela. Lugo, España. 203 p. 